\title{
The Relaxation Oscillation of Turbulent Convection in Rotating Cylindrical Annulus
}

\author{
J. Tao
}

LTCS and Department of Mechanics and Aerospace Engineering, Peking University, Beijing 1000871, China

Email: jjtao@pku.edu.cn

\begin{abstract}
In the last decades two-dimensional turbulence developing in strongly anisotropic systems such as magnetic confinement devices and fast rotating geophysical fluids has been the subject of great concern because of their complex transport phenomena, and the main features are the bursts in the fluctuation level and relaxation oscillations in the kinetic energy[1 3]. The mechanism underlying relaxation-oscillation generation and stability in turbulent convection driven by centrifugal buoyancy is studied in this paper. At high Rayleigh number, the vacillating unstable mode subdues to a new secondary vacillation instability, whose standing-wave type oscillation in the azimuthal direction leads to the large-scale intermittency, the chaotic relaxation oscillation. It is illustrated that the relaxation oscillation can only be triggered when the dominant wave number lies in a narrow range due to the strong shear stress exerted by the no-slip boundary, and the Coriolis effect on its generation is also discussed.
\end{abstract}

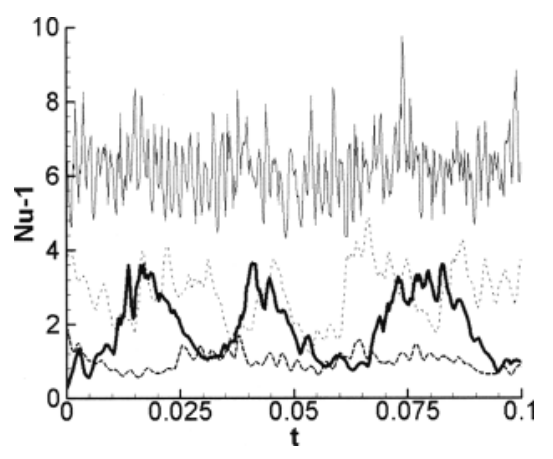

Figure: The time evolution of Nusselt number as a function of the Coriolis parameter $\eta$ and Rayleigh number $R a$. The thick solid line, dash line and the thin solid line correspond to $\eta=42000,3000(\mathrm{l}$ and 10000 for $R a=4500000$, respectively. The bottom long dash line is calculated for $\eta=42000$ and $R a=2900000$ as a reference

\section{REFERENCES}

1. Brummell N.H., Hart J.E. High Rayleigh number $\beta$-convection. Geophys. Astrophys. Fluid Dyn. 1993:68:85

2. Busse F.H. Convective flows in rapidly rotating spheres and their dynamo action. Phys.Fluids, 2002; 14:1301

3. Busse F.H., Clever R.M. Bursts in inclined layer convection. Phys.Fluids, 2000;12:2137 